\title{
Quality of Life in Patients with Mild or Moderate Acne Vulgaris
}

\author{
Coban $\mathbf{M}^{1}$, Turkmen $\mathbf{M}^{1}$ and Coban $\mathrm{K}^{2}$ \\ 1Izmir Bozyaka Training and Research Hospital, Turkey \\ ${ }^{2}$ Dr. Behcet Uz Children's hospital, Turkey
}

*Corresponding author: Melis Coban, Izmir Bozyaka Training and Research Hospital, Turkey, Tel: 2322505050; E-mail: drmelis@gmail.com

\section{Research Article \\ Volume 2 Issue 3}

Received Date: August 01, 2017

Published Date: August 18, 2017

DOI: $10.23880 /$ cdoaj16000129

\section{Abstract}

Acne vulgaris is a dermatological genetic-hormonal, chronic inflammatory illness, self-limited, in pilosebaceous locations, with formation of comedones, papules and cysts in which evolution to a greater inflammatory process is added, leading to formation of pustules and abscesses, with frequent cicatricial success, causing great psychological impact in patients affected by this disease. There are several acne-specific health related quality of life instruments; one of them is acne quality of life scale. There are a small number of studies about acne quality of life scale in literature. In our study, we aim to have a reliable scientific data about this subject and contribute to the literature. We used the first dimension of AQOLS for our study, social quality-quality of life. One hundred seventy-two patients were enrolled in this study. We have found correlation between female gender and SOCQOL. According to this result, we think that acne affects life quality of women more effective than men. We found a negative correlation between SOCQOL and age. When the age increases, SOCQOL decreases. We think about that so, when the patients get older, they get used to their acne lesions and SOCQOL scores decrease. There was correlation between pretreatment and SOCQOL. This means that life quality of a patient with acne decreases if the number of pretreatments increases.

As the clinician determines the treatment, acne severity of the patient is important, but nowadays the defining of acne quality of life becomes more important for determining the method of the treatment. There are some tests for this. SOCQOL is the most useful test between them. We think that our study provides useful results for the literature. Additionally, we think a clinician shouldn't neglect the psychological statement of patients with acne as he/she determines the method of treatment.

Keywords: Acne Vulgaris; Quality of life

Abbreviations: HRQOL: Health Related Quality of Life; ADI: Acne Disability Index; CADI: Cardiff Acne Disability Index; CADI: Cardiff Acne Disability Index; AQOLS: Acne Quality Of Life Scale; acne-QOLI: Acne Quality of Life Inventory; ASIS: Acne Symptom and Impact Scale; acne-QOL: Acne Specific-Quality of Life; SOCQOL:
Social Impact of Acne; VOCQOL: Vocational Impact of Acne; GAGS: Global Acne Grading System.

\section{Introduction}

Acne vulgaris is a dermatological genetic-hormonal, chronic inflammatory illness, self-limited, in 
pilosebaceous locations, with formation of comedones, papules and cysts in which evolution to a greater inflammatory process is added, leading to formation of pustules and abscesses, with frequent cicatricial success, causing great psychological impact in patients affected by this disease [1]. The most severe forms of acne vulgaris occur more frequently in males, but the disease tends to be more persistent in females [2]. Acne vulgaris lesions predominate especially on face and thorax, which leads to feelings of guilt, shame and social isolation [1,3]. Acne vulgaris isn't a life-threatening disease; however, it may cause anxiety, depression, anger, suicidal thoughts and body dysmorphic disorder [4]. The levels of social, psychological and emotional impairments in acne is comparable with chronic diseases such as asthma, epilepsy, diabetes and arthritis [1,2]. There are several acne-specific health related quality of life (HRQOL) instruments, for example, including the assessment of the psychological and social effects of acne (APSEA), the acne disability index (ADI), the Cardiff acne disability index (CADI) and acne quality of life scale (AQOLS) [2-5]. Also, acne quality of life inventory (acne-QOLI), acne symptom and impact scale (ASIS) and acne specific-quality of life (acne-QOL) are other scales for acne vulgaris. AQOLS was founded by Gupta et al in 1998 and focuses more on the individual handicap associated with acne [6,7]. The scale consists of 12 items and takes into account two dimensions of quality of life. The first dimension consists of a nine-item questionnaire that addresses the social impact of acne (SOCQOL). The second dimension consists of a three-item questionnaire that addresses the vocational impact of acne (VOCQOL) [7]. We used SOCQOL for our study because we wanted to understand the psychological effects of acne vulgaris and there are a small number of studies about acne quality of life determined with SOCQOL about Turkish people in literature. In our study, we aim to have a reliable scientific data about this subject and contribute to the literature.

\section{Materials and Methods}

\section{Patients}

We included the patients, aged 15-40 who came to the dermatology polyclinic of Bozyaka Research and Training Hospital between April 2016 and May 2017. The patients signed the informed consent form and this form was signed by the parents of the patients who are lower than 18 years old. We asked them about their age, their gender, duration of acne and their previous treatments. After that, we examined them. We noted the places where acne occurred on skin (face, back, chest), calculated global acne grading system (GAGS) score and AQOLS of each patient. We included only patients with mild or moderate acne.
GAGS and AQOLS were calculated by the same dermatologist.

\section{GAGS}

The clinical severity of acne was assessed using the global acne grading system score which calculates global score by adding the individual scores of six locations, five on the face (forehead, right and left cheek, nose and chin) and the sixth, a combined one for chest and upper back, each location having already been assigned a fixed score based on the surface area, distribution and density of pilosebaceous units. A score of 1-18 is considered as mild; 19-30 as moderate; $31-38$ as severe and more than 39 as very severe [8].

\section{AQOLS}

Acne quality of life scale was founded by Gupta et al in 1998 an focuses more on the individual handicap associated with acne [6,7]. The scale consists of 12 items and takes into account two dimensions of quality of life. The first dimension consists of a nine-item questionnaire that addresses the social impact of acne. The second dimension consists of a three-item questionnaire that addresses the vocational impact of acne [7]. This scale also appears to be useful for evaluating anxiety and depression and aims to determine the relationship between the acne severity and the psychological morbidity that occurred because of acne and has proved its reliability and validity (Cronbach alpha $=0.94, \mathrm{r}=$ 0.99). It can be used only for patients with mild or moderate acne vulgaris. It is instructed to answer each question in the form of not at all $=0$, mildly $=1$, moderately $=2$, very markedly $=3$. The total score is obtained by adding up the answers to each question. When the score is equel to nine or higher than nine, it is commented as high score. When it is lower than nine, it is commented as low score [7,9]. We used the study of Demircay, et al. while we asked the questions of the first dimension of SOCQOL as Turkish version. When the score is less than nine, it is determined as low score. When the score is equal to nine or more than nine, it is determined as high score [10].

\section{Statistical Analysis}

Categorical variables were summarized in terms of counts and relative frequencies. Numeric variables were summarized in terms of man \pm SD (standard deviation). We used Spearman correlation test to examine relationship between GAGS and AQOLS, between acne duration and AQOLS, between age and AQOLS. T test was used to examine relationships between other variables. 
All data were analysed using SPSS version 17.0 for Windows. $\mathrm{P}<0.05$ was deemed statistically significant.

\section{Contribution}

Melis Çoban contributed to the planning and writing of the study. Kazım Çoban contributed to the statistical analysis and writing of the study. Meltem Turkmen conduced to writing of the study.

\section{Ethical Consideration}

This study was conducted in accordance with the ethical principles derived from the Declaration of Helsinki and on Harmonization Good Clinical Practices and in compliance with local regulatory requirements and was reviewed and approved by the local ethics committees for these institutions. All patients provided their written informed consent before entering the study.

\section{Results}

One hundred seventy-two patients were enrolled in this study. There were 115 women and 57 men and the mean age at diagnosis was 19.66 \pm 2.93 years (min 15 years, max 32 years). The mean acne duration was $4.08 \pm 2.71$ years (min 1 year, max 14 years). We determined that 124 patients had previous treatment and 48 patients didn't have any previous treatment. 2 patients didn't have any acne lesion on face, 90 patients didn't have any acne on body, 80 patients had acne both on their face and their body. The mean GAGS were 17.42 \pm 5.29 (min 1, max 29). 98 patients of them were in the mild group and 74 patients of them were in the moderate group. The mean AQOLS was 2.58 \pm 2.82 (min 0, max 12). 163 patients of them were in low score group and 9 patients of them were in high score group (Table 1).

We found no significant difference between GAGS and AQOLS $(p=0.2)$, between acne duration and AQOLS $(\mathrm{p}=0.7)$, between acne on face and AQOLS $(\mathrm{p}=0.06)$, acne on face-body and AQOLS ( $\mathrm{p}=0.6)$, between acne on body and AQOLS $(\mathrm{p}=0.4)$, between gender and GAGS $(\mathrm{p}=0.09)$.

There was significant correlation between female gender and AQOLS $(p=0.046)$. On this basis, we determined that the psychological impact of acne is more effective on female patients. We found significant correlation between the previous treatment and AQOLS $(\mathrm{p}=0.04)$. This proved that psychological impact of acne is more effective on the patients who had used some previous treatments. The study shows that there was significant correlation between age and AQOLS $(\mathrm{p}=0.006$, $\mathrm{r}=-0.2$ ). According to this finding, when the patients get older, AQOLS scores get higher (Table 2).

\begin{tabular}{|c|c|}
\hline Age (years) & \\
\hline Mean age & 19.66 \\
\hline Age range & $15-32$ \\
\hline Gender, n (\%) & $115(66.8 \%)$ \\
\hline Female & $57(33.2 \%)$ \\
\hline Male & 4.08 \\
\hline Previous treatment, n (\%) & \\
\hline Yes & $124(72 \%)$ \\
\hline No & $48(28 \%)$ \\
\hline mocne duration (years) & \\
\hline Only face & $90(52.3 \%)$ \\
\hline Only body & $2(1.2 \%)$ \\
\hline Both face and body & $80(46.5 \%)$ \\
\hline Severity of acne, n (\%) & \\
\hline Mild & $98(56.9 \%)$ \\
\hline Moderate & $74(43.1 \%)$ \\
\hline SocQoL score, n (\%) & $163(94.7 \%)$ \\
\hline Low & $9(5.3 \%)$ \\
\hline High & \\
\hline
\end{tabular}

Table 1: Demographic data of patients and features of lesions.

\begin{tabular}{|c|c|c|c|}
\hline & n & Mean \pm Std & p \\
\hline \multicolumn{3}{|c|}{ Gender } \\
\hline Female & 115 & $16.95 \pm 5.2$ & 0.046 \\
\hline Male & 57 & $18.39 \pm 5.2$ & 0.065 \\
\hline \multicolumn{4}{|c|}{ Previous treatment } \\
\hline Yes & 124 & $2.84 \pm 2.9$ & 0.04 \\
\hline No & 48 & $1.92 \pm 2.4$ & 0.55 \\
\hline \multirow{2}{*}{ Age } & & $19.66 \pm 0.2$ & 0.006 \\
\cline { 3 - 4 } & & $\mathrm{r}=-0.2$ \\
\hline
\end{tabular}

Table 2: AQOLS average scores of study group according to different variables 


\section{Discussion}

One of the diseases that affect life quality is acne vulgaris [5]. According to literature, acne vulgaris has a high frequency, with a prevalence varying between $39.9 \%$ and $96 \%$, depending on the age bracket and the population studied [11]. Studies indicate that up to $95 \%$ of men and $83 \%$ of women are affected by it until they reach their twenties. In approximately $10 \%$ of cases, acne becomes severe [5].

The relationship between dermatologic and psychiatric disorders has been of interest since at least 1891, with the description by Brocq and Jacquet of "neurodermatitis". Several studies on psychologic characteristic of patients with acne vulgaris have reported diminished "general well being", negative self-image, increased anxiety and depression, reduced self-esteem and social withdrawal relative to on-dermatologic normal populations [7].

One of the tests which examine the effects of acne vulgaris on quality of life is SOCQOL of AQOLS. This test has appropriate subtitles which can determine the effects about social relationships of the patient. In addition, it is coherent with psychological tests [10]. It is focused on individual handicap, is useful for evaluating anxiety and depression of patient, is validated in mild or moderate acne and has no difference between men and women. There are some quality of life tests for acne, but AQOLS appears to be the most useful one [7].

We have found no relationship between SOCQOL and acne severity. However, acne is known to have psychological effects on people [9]. This result supports Medansky who has suggested that acne may not have a major emotional impact on the patient [12]. In the study of Ilgen, et al. with 108 patients, Basak et al. with 176 patients, Kaymak, et al. with 84 patients and Yazıci, et al. with 99 patients there was no relationship between acne severity and SOCQOL, too $[5,12,13]$. We think that acne severity don't affect the social life of the patients. Whatever the result of GAGS is, the patients can keep going their relationship with other people. According to Mallon et al. there is no correlation between acne severity and life quality because the individual, social, cultural and traditional society of the patient determines the severity of acne-induced psychological problems [14]. SOCQOL isn't enough alone for the effects of acne severity. According to Al-Shidhani, et al. any increase in acne severity would lead to an increase in the negative effects on the patients' feelings, self-perception, and socialization as the lesions became more prominent and if scarring occurred [15]. Few studies reported a significant relationship between the severity of acne and its effect on the patient's QoL $[16,17]$. Additional psychological measurements should be used because there are different results about the relationship between acne severity and AQOLS.

Further, there was no relationship between acne duration, age and SOCQOL in these two studies, but in our study we found a negative correlation between SOCQOL and age. When the age increases, SOCQOL decreases. We think about that so, when the patients get older, they get used to their acne lesions and SOCQOL scores decrease.

We have found no correlation between acne duration and SOCQOL, as reported Ilgen, et al. Just the opposite, Kaymak, et al found relationship between acne duration and SOCQOL $[12,5]$. Interestingly, we also didn't found any relationship between acne on face, acne on body, acne on face-body and SOCQOL. According to this result, the body part where acne is, don't affect quality of life. We think that acne don't disturb the patients. Maybe, they have some problems more important than acne in their social life or academic life. This can be a topic for another study.

We have found correlation between female gender and SOCQOL. According to this result, we think that acne affects life quality of women more effective than men. According to Yazıcl, et al. and Kaymak, et al., there is no relationship between gender and SOCQOL [13,5]. They think that gender doesn't effect acne quality of life [5]. However, Al-Shidhani, et al. think that female patients are more negatively affected than males, as expected, according to their study [15]. The same results are seen in other studies [18-21]. This difference is not surprising, as females are usually more concerned about their physical appearance.

There was correlation between pretreatment and SOCQOL. This means that life quality of a patient with acne decreases if the number of pretreatments increases. The patients fatigue between various treatments and want more effective solutions about their acne problem.

\section{Conclusion}

As the clinician determines the treatment, acne severity of the patient is important, but nowadays the defining of acne quality of life becomes more important for determining the method of the treatment. There are some tests for this. AQOLS which has two dimensions, is the most useful test between them.

AQOLS examines the relation between acne severity and quality of life. This scale needs to be tested among 
other patient samples representing both sexes before its generalizability can be confirmed. When using this scale, it may not be necessary to administer other psychological instruments because its scores correlate significantly with wide range psychopathologic measures. It appears that AQOLS is a useful test for examination the psychological statements of patients with mild or moderate acne vulgaris.

According to our study, acne affects especially female patients. Life quality of a patient with acne decreases if the number of pretreatments increases. When the patients get older, SOCQOL scores get higher.

We think that our study provides useful results for the literature. Additionally, we think a clinician shouldn't neglect the psychological statement of patients with acne as he/she determines the method of treatment.

\section{References}

1. Vilar GN, Santos LA, Filho JFS (2015) Quality of life, self-esteem and psychosocial factors in adolescents with acne vulgaris. An Bras Dermatol 90(5): 622-629.

2. Nair PA, Nair AR (2015) Quality of Life Perspective Towards Acne among Adolescents at Tertiary Care Center of Gujarat, India. J Clin Diagn Res 9(10): WC01WC04.

3. Gieler U, Gieler T, Kupfer JP (2015) Acne and quality of life - impact and management. J Eur Acad Dermatol Venereol 29(4): 12-14.

4. Bağcioğlu E, Bahçeci B, Öztürk A, Deveci E, Şaşmaz S, et al. (2014) Quality of life, problem solving, focus of control and anger tendency in the patients with acne. Türkderm 48(4): 177-781.

5. Kaymak Y, Adışen E, Çelik B, Gürer MA (2007) Effects of mild to moderate acne vulgaris on quality of life. Turkiye Klinikleri J Dermatol 17: 99-104.

6. Gupta MA, Johnson AM, Gupta AK (1998) The development of an acne quality of life scale: reliability, validity, and relation to subjective acne severity in mild to moderate acne vulgaris. Acta Derm Venereol 78(6): 451-456.

7. Dreno B (2006) Assessing Quality of life in patients with acne vulgaris, implications for treatment. Am J Clin Dermatol 7(2): 99-106.

8. Gupta A, Sharma YK, Dash KN, Chaudhari ND, Jethani S (2016) Quality of life in acne vulgaris: Relationship to clinical severity and demographic data. Indian J Dermatol Venereol Leprol 82(3): 292-397.

9. Ilgen E, Aytimur D (2005) There is no correlation between acne severity and AQOLS/DLQI scores. The Journal of Dermatology 32(9): 705-710.

10. Demirçay Z, Şenol A, Seçkin D, Demir F (2006) Reliability of Turkish version of acne quality of life scale in patients with acne vulgaris. Türkderm 40(3): 94-97.

11. Costa A, Alchorne MMA, Goldschmidt MCB (2008) Etiopathogenic features of acne vulgaris. An Bras Dermatol 83(5): 451-159.

12. Medansky RS, Handler RM, Medansky DL (1981) Selfevaluation of acne and emotion: a pilot study. Psychosomatics 22(5): 379-383.

13. Yazici K, Baz K, Yazıcı AE, Kokturk A, Tot S, et al. (2004) Disease-specific quality of life is associated with anxiety and depression in patients with acne. J Eur Acad Dermatol Venereol 18(4): 435-439.

14. Mallon E, Newton JN, Kalssen A, Stewart-Brown SL, Ryan TJ, et al. (1999) The quality of life in acne: a comparison with general medical conditions using generic questionnaries. Br J Dermatol 140(4): 672676.

15. Al-Shidhani A, Al-Rashdi S, Al-Habsi H, Rizvi S (2015) Impact of Acne on Quality of Life of Students at Sultan Qaboos University. Oman Med J 30(1): 42-47.

16. Klassen AF, Newton JN, Mallon E (2000) Measuring quality of life in people referred for specialist care of acne: comparing generic and disease-specific measures. J Am Acad Dermatol 43(2 Pt 1): 229-233.

17. Shahzad N, Nasir J, Ikram U, Asmaa-ul-Haque, Qadir A, et al. (2011) Frequency and psychosocial impact of acne on university and college students. J Coll Physicians Surg Pak 21(7): 442-443.

18. Pearl A, Arroll B, Lello J, Birchall NM (1998) The impact of acne: a study of adolescents' attitudes, perception and knowledge. N Z Med J 111(1070): 269-271.

19. Merck, Co Inc (1997) Acne-Specific Quality of Life Questionnaire (Acne-QoL): Manual and Interpretation Guide. Whitehouse Station, NJ Merck and Co Inc. 
20. Rapp DA, Brenes GA, Feldman SR, Fleischer AB Jr, Graham GF, et al. (2004) Anger and acne: implications for quality of life, patient satisfaction and clinical care. Br J Dermatol 151(1): 183-189.
21. Smithard A, Glazebrook C, Williams HC (2001) Acne prevalence, knowledge about acne and psychological morbidity in mid-adolescence: a community-based study. Br J Dermatol 145(2): 274-279. 